\title{
Blocking Hepatoma-Derived Growth Factor Attenuates Vasospasm and Neuron Cell Apoptosis in Rats Subjected to Subarachnoid Hemorrhage
}

\author{
Chia-Li Chung ${ }^{1,2} \cdot$ Chieh-Hsin $\mathrm{Wu}^{3,4} \cdot$ Yu-Hua Huang ${ }^{5} \cdot$ Shu-Chuan Wu ${ }^{3} \cdot$ Chee-Yin Chai ${ }^{6,7} \cdot$ Hung-Pei Tsai ${ }^{3,8}$ (D) \\ Aij-Lie Kwan ${ }^{3,4,8,9}$
}

Received: 12 May 2021 / Revised: 21 June 2021 / Accepted: 25 June 2021 / Published online: 5 July 2021

(c) The Author(s) 2021

\begin{abstract}
Subarachnoid hemorrhage (SAH) is an important subcategory of stroke due to its unacceptably high mortality rate as well as the severe complications it causes, such as cerebral vasospasm, neurological deficits, and cardiopulmonary abnormality. Hepatoma-derived growth factor (HDGF) is a growth factor related to normal development and is involved in liver development and regeneration. This study explored the relationship between SAH and HDGF. Sixty rats were divided into five groups ( $\mathrm{n}=12$ /group): (A) control group; (B) rHDGF ab only group [normal animals treated with $50 \mu \mathrm{M}$ recombinant HDGF antibodies (rHDGF ab)]; (C) SAH group; (D) SAH + pre-rHDGF ab group (SAH animals pre-treated with $50 \mu \mathrm{M}$ rHDGF $\mathrm{ab}$ into the subarachnoid space within $24 \mathrm{~h}$ before SAH); and (E) SAH + post-rHDGF ab group (SAH animals post-treated with $50 \mu \mathrm{M}$ rHDGF ab into the subarachnoid space within $24 \mathrm{~h}$ after SAH). At $48 \mathrm{~h}$ after SAH, serum and cerebrospinal fluid (CSF) samples were collected to measure the levels of pro-inflammatory factors by ELISA, and rat cortex tissues were used to measure protein levels by western blot analysis. Immunofluorescence staining for Iba-1, GFAP, TUNEL, and NeuN was detected proliferation of microglia and astrocyte and apoptosis of neuron cells. Neurological outcome was assessed by ambulation and placing/stepping reflex responses. Morphology assay showed that pre-treatment and post-treatment with rHDGF ab attenuated vasospasm after SAH. SAH up-regulated the levels of TNF- $\alpha$, IL- $1 \beta$, and IL- 6 in both the CSF and serum samples, and both pre- and post-treatment with rHDGF ab inhibited the up-regulation of these pro-inflammatory factors, except for the serum IL-6 levels. Western blot analysis demonstrated that SAH up-regulated pro-BDNF and NFkB protein levels, and both pre- and post-treatment with rHDGF ab significantly reduced the up-regulation. The result from immunofluorescence staining showed that SAH induced proliferation of microglia and astrocyte and apoptosis of neuron cells. Both pre- and post-treatment with rHDGF ab significantly attenuated proliferation of microglia and astrocyte and inhibited apoptosis of neuron cells. Furthermore, treatment with rHDGF ab significantly improved neurological outcome. Blocking HDGF attenuates neuron cell apoptosis and vasospasm through inhibiting inflammation in brain tissue at early phase after SAH.
\end{abstract}

\section{Introduction}

Early brain injury that occurs at the time of bleeding is the leading cause of mortality (30-70\%) following subarachnoid hemorrhage (SAH) [1, 2]. SAH survivors are at risk of developing delayed cerebral vasospasm, delayed cerebral

\footnotetext{
Hung-Pei Tsai

carbugino@gmail.com

Aij-Lie Kwan

aijliekwan@yahoo.com.tw
}

Extended author information available on the last page of the article ischemia, or delayed ischemic neurological deficits during the hospital course [2]. Delayed vasospasm develops in approximately $70 \%$ of patients between 3 and 14 days after SAH [1, 2]. For decades, it has been considered as the single and the most important cause of delayed cerebral ischemia and poor outcome [3]. Even patients with favorable outcomes are frequently left with significant residual memory, reduced executive functioning, or language deficits [4]. Cerebral vasospasm following aneurysmal SAH is the leading cause of death and disability after aneurysm rupture [5]. Cerebral ischemia secondary to vasospasm occurs in 20 to $30 \%$ of these patients and has been correlated with a 1.5 to threefold increase in mortality in the first 2 weeks after 
SAH [3, 6]. Although cerebral vasospasm associated with SAH has been recognized for more than 50 years, adequate treatment is still illusive. Thence, the pathophysiological mechanism contributing to this form of arterial dysfunction is a topic of intense experimental study.

One of the major consequences of early brain injury is apoptosis, which is known to occur within minutes to $24 \mathrm{~h}$ after SAH [7]. Immune cells, including microglia and astrocytes, release pro-inflammatory factors to induce cell apoptosis and activation of transcription factor, resulting in positive feedbacks. Microglia and astrocytes also mediate neuropathic behavior by modulating the activity of spinal neurons to cause central sensitization, which has been associated with inflammatory neuropathies in autoimmune thyroid diseases, complex regional pain syndrome, osteoarthritis, rheumatoid arthritis, post-operative pain, and SAH [7, 8].

Hepatoma-derived growth factor (HDGF) is a 240 aminoacid protein isolated from human hepatoma cells [9]. Surface expressed nucleolin has recently been identified as an HDGF receptor [10]. During cell development, HDGF stimulates cell proliferation in fibroblasts, endothelial cells, and hepatoma cells [11]. It is also a growth factor related to tissue organogenesis and is involved in the development and regeneration of the liver [12-14], lungs [15, 16], kidney [17], heart [18], and the vascular system [19-21]. On the other hand, HDGF expression is associated with various malignant cancers, including hepatocellular carcinoma (HCC), gastric cancer, non-small cell lung cancer, pancreatic cancer, and melanoma [22, 23], to name a few. In addition, HDGF plays important roles in various cellular events, including ribosome biogenesis, RNA processing, DNA damage repair, and transcriptional regulation [17]. Furthermore, many studies indicate that HDGF is a mitogen with extracellular proliferative effects on hepatoma cells, fibroblasts, vascular smooth muscle cells, and endothelial cells [16]. However, no reports showed the relationship between HDGF and SAH. Hence, this was the focus of the present investigation.

\section{Materials and Methods}

\section{Animal Preparation}

The study procedures were executed in accordance with the protocol approved by the Committee of Institutional Animal Research at Kaohsiung Medical University. Male Sprague-Dawley rats were purchased from BioLasco (BioLasco Taiwan Co., Ltd., Taipei, Taiwan, authorized by Charles River Lab). After arriving at the Kaohsiung Medical University vivarium, the rats were acclimated for at least 1 week before being used in the experiment. All rats were housed at a constant temperature $\left(24^{\circ} \mathrm{C}\right)$ and at regular light/ dark cycles between 6:00 am and 6:00 pm, with free access to a standard diet. Animals weighing between 350 and $450 \mathrm{~g}$ were used in this study.

\section{SAH Induction}

The one-shot SAH model was utilized. Briefly, rats were anesthetized by intraperitoneal injection of $40 \mathrm{mg} / \mathrm{kg}$ Zoletil 50® containing a mixture of zolazepam and tiletamine hypochloride (Virbac, Carros, France). The head was fixed in a stereotactic apparatus (Stoelting, Wood Dale IL, USA), and a 25 -gauge butterfly needle was advanced into the cisterna magna to withdraw $0.3 \mathrm{~mL}$ of cerebrospinal fluid (CSF). Fresh, autologous, and non-heparinized blood $(0.1 \mathrm{~mL} / 100 \mathrm{~g}$ of body weight $)$ drawn from the central tail artery was then slowly instilled into the subarachnoid space through a butterfly needle and tubing. Afterwards, the animals were kept in a ventral recumbent position for at least 30 min to promote ventral blood distribution. The morbidity caused by SAH was $100 \%$, and the mortality was $0 \%$ in this study. The respiratory pattern of rats was inspected closely, and mechanical ventilation was provided if necessary. Upon fully awakening, the animals were sent back to the vivarium. In this study, there are five groups: (1) control group; (2) rHDGF ab group, treatment with rHDGF antibody into the subarachnoid space; (3) SAH group; (4) SAH + pre-rHDGF ab group, treatment with rHDGF antibody into the subarachnoid space within $24 \mathrm{~h}$ before $\mathrm{SAH}$; and (5) $\mathrm{SAH}+$ postrHDGF ab group, treatment with rHDGF antibody into the subarachnoid space within $24 \mathrm{~h}$ after SAH. All rats were used following randomization.

\section{Preparation of HDGF Hyperimmune Serum (Recombinant HDGF Antibody)}

HDGF hyperimmune serum was customized by Leadgene Biomedical Inc. (Tainan, Taiwan). Briefly, 6- to 8-week-old $\mathrm{BALB} / \mathrm{c}$ mice were primed by intraperitoneal injection of $50 \mu \mathrm{g}$ of recombinant rat HDGF (rHDGF) protein in complete Freund's adjuvant. Two weeks after the first injection, the mice were given another injection of $25 \mu \mathrm{g}$ rHDGF antibody in PBS. The procedure was repeated at weeks 4 and 5 after antigen priming. The sera were collected and stored at $-20{ }^{\circ} \mathrm{C}$ until use.

\section{Neurological Evaluation}

We followed the method of neurobehavioral evaluation from Dr. Huang et al. (2017) [24]. Neurobehavioral evaluation of animals was performed by assessing the sensorimotor integration of the forelimb and hind-limb activities using a modified limb-placing test that consisted of ambulation as well as placing and stepping reflex [25]. Motor deficit index (MDI) represented the sum of scores for walking by lower 
limbs and for placing/stepping response and was determined at $48 \mathrm{~h}$ after induction of SAH. Higher MDI values indicated poorer neurological outcomes. In addition, neurological evaluation was used as a double-blind trial.

\section{Tissue Processing}

This protocol was conducted in a as previously described in Dr. Huang et al. (2017) [24]. At the end of experiments, each animal was anesthetized again for perfusion and fixation. The thoracic cage was opened, and the left ventricle was canalled using a No. 16 catheter. After clamping, the descending aorta and puncturing the right atrium, the brain was perfused with $180 \mathrm{~mL}$ of $2 \%$ paraformaldehyde and then $100 \mathrm{~mL}$ of phosphate buffer $(0.01 \mathrm{M})$ under $36^{\circ} \mathrm{C}$ and $100 \mathrm{~mm} \mathrm{Hg}$ perfusion pressure. Gross inspection of harvested brains was performed to confirm the presence of subarachnoid blood clots over the basilar artery (BA), and the specimen was immersed in a fixative solution. The BAs were then separated from the brainstems, and the middle third of each vessel was dissected out. These arterial segments were flat-embedded in paraffin, and BA cross sections were cut into $3-\mu \mathrm{m}$ sections and were stained with hematoxylin and eosin stain for subsequent analysis.

\section{Morphometric Assessment of BA}

This protocol was conducted in a as previously described in Dr. Huang et al. (2017) [24]. Three cross sections from the middle-third BA in each animal were analyzed by a trained research staff blinded to the experimental groups. The thickness of BA was defined as the largest vertical distance between the inner surface of endothelium and the outer surface of adventitia. The arterial cross-sectional area was calculated using computer-based morphometric analysis (Image J; Universal Imaging Corp., USA). The average area of BA cross sections from each rat was calculated to obtain mean values for the degree of vasospasm at $48 \mathrm{~h}$ after SAH.

\section{Immunofluorescence Staining}

After deparaffinization and rehydration, paraffin-embedded brain samples were heated by steam for $30 \mathrm{~min}$ to retrieve antigen using DAKO antigen retrieval solution (DAKO, Carpenteria, CA). Slides were then washed twice with Trisbuffered saline (TBS) and immersed in a 3\% hydrogen peroxide solution for $10 \mathrm{~min}$ to inhibit endogenous peroxidase. Upon washing twice in TBS, the sections were incubated with TUNEL kit, mouse anti-NeuN (Merck; MAB377; Germany), mouse anti-GFAP (Sigma; G3893; USA), and rabbit anti-Iba1 (proteintech; 10,904-1-AP; USA) antibodies at room temperature to detect the presence of the cell number of DNA damage, astrocytes, microglia, and neuron cells, respectively. Slides were again washed twice with TBS and subsequently incubated with anti-rabbit antibody (Thermo; A11008; USA) and anti-mouse antibody (Thermo; A10524; USA) for $3 \mathrm{~h}$ at room temperature. Afterwards, the slides were washed twice with TBS, stained and mounted within Fluroshield ${ }^{\mathrm{TM}}$ with DAPI (Sigma; F6057; USA), and take a picture following LSM 700 confocal microscope.

\section{Western Blot}

Rat cortex samples were collected at $48 \mathrm{~h}$ after SAH. Tissue extracts were prepared in $1 \mathrm{~mL}$ of ice-cold lysis buffer [50 mM Tris-HCl, pH 7.4, 0.25 M NaCl, 0.1\% Nonidet P-40, $5 \mathrm{mM}$ EDTA, $50 \mathrm{mM} \mathrm{NaF}, 1 \times$ cocktail of protease inhibitors (Sigma, St. Louis, MO), $1 \mathrm{mM}$ phenylmethylsulfonyl fluoride, and $1 \mathrm{mg} / \mathrm{L}$ aprotinin] and incubated on ice for $30 \mathrm{~min}$. After centrifugation at $8000 \mathrm{~g}$ for $20 \mathrm{~min}$ at $4{ }^{\circ} \mathrm{C}$, protein amount in the supernatant was quantified using a protein assay kit from Bio-Rad Laboratories, and $60 \mu \mathrm{g}$ of protein samples were separated by $12 \%$ SDS-polyacrylamide gel electrophoresis. Proteins were then transferred to nitrocellulose membranes. The membranes were blocked in PBS containing 5\% fat-free milk for $90 \mathrm{~min}$ at room temperature and then incubated with antibodies (HDGF and cleaved caspase-3) for $2 \mathrm{~h}$ at room temperature. After washing, the membranes were incubated for $1 \mathrm{~h}$ at $25^{\circ} \mathrm{C}$ with the appropriate horseradish peroxidase-labeled secondary antibodies, and the bound antibodies were visualized and quantified by chemiluminescence detection. The expression level of proteins of interest was normalized to the densitometric units of $\beta$-actin.

\section{ELISA}

Serum and cerebrospinal fluid (CSF) samples were collected at $48 \mathrm{~h}$ after $\mathrm{SAH}$, centrifuged immediately at $2000 \times g$ for $10 \mathrm{~min}$ at $4{ }^{\circ} \mathrm{C}$ to remove cells, and were stored below $-15^{\circ} \mathrm{C}$ prior to analysis. $\mathrm{X}$ These samples were concentrated by passing through $\mathrm{C} 2$ columns (Amersham, Nutley, USA), and the levels of TNF- $\alpha$, IL- $1 \beta$, and IL- 6 were determined using an inflammatory factor ELISA system (Amersham) at $450 \mathrm{~nm}$.

\section{Statistics}

The results were analyzed using SPSS version 20.0 (IBM SPSS Statistics, location). Data were presented as mean \pm standard deviation (SD). Group results were compared using Student's $t$ test, Mann-Whitney $U$ test, or oneway analysis of variance (ANOVA). A $P$ value of $<0.05$ was considered statistically significant. 


\section{Results}

\section{Neurological Outcomes}

The neurobehavioral scores, including ambulation, placing/stepping reflex, and MDI, were not different between the control and rHDGF ab only groups (Table 1). In animals subjected to SAH, both the ambulation $(1.75 \pm 0.46)$ and placing/stepping reflex $(1.88 \pm 0.35)$ scores were

Table 1 Behavioral assessment

\begin{tabular}{llll}
\hline Treatment & Ambulation & $\begin{array}{l}\text { Placing/stepping } \\
\text { reflex }\end{array}$ & MDI \\
\hline Control & 0 & 0 & 0 \\
rHDGF ab & 0 & 0 & 0 \\
SAH & $1.75 \pm 0.46$ & $1.88 \pm 0.35$ & $3.63 \pm 0.74$ \\
SAH+ pre-rHDGF & $1.12 \pm 0.64^{*}$ & $1.25 \pm 0.71^{*}$ & $2.38 \pm 1.19^{*}$ \\
$\quad$ ab & & & \\
$\begin{array}{l}\text { SAH+ post-rHDGF } \\
\quad 1.38 \pm 0.52^{*}\end{array}$ & $1.25 \pm 0.46^{*}$ & $2.63 \pm 0.74^{*}$ \\
$\quad$ & & &
\end{tabular}

Neurological outcome was assessed by ambulation and placing/stepping reflex responses within $48 \mathrm{~h}$ after inducing SAH. MDI, motor deficit index, which is the sum of ambulation (walking with lower extremities) and placing/stepping reflex (dragging the dorsum of hind paw over the edge of a surface) scores significantly higher than the non-SAH animals. Pre-treatment with rHDGF ab significantly decreased both the ambulation $(1.12 \pm 0.64 ; \mathrm{P}<0.05)$ and the placing/stepping reflex $(1.25 \pm 0.71)$ scores when compared with the SAH group. In addition, post-treatment with rHDGF ab significantly also decreased both the ambulation $(1.38 \pm 0.52 ; \mathrm{P}<0.05)$ and the placing/stepping reflex $(1.25 \pm 0.46)$ scores when compared with the SAH group. Likewise, MDI in the rHDGF ab pre-treatment group $(2.38 \pm 1.19 ; \mathrm{P}<0.05)$ and post-treatment group $(2.63 \pm 0.74 ; \mathrm{P}<0.05)$ were also significantly reduced when compared with the SAH group $(3.63 \pm 0.74)$ (Table 1).

\section{Morphological Changes in BA}

Upon microscopic examination, endothelial deformation, twisting of internal elastic laminae, and smooth muscle necrosis were seen in the BAs of rats subjected to $\mathrm{SAH}$ (compare Fig. 1C, D and E with Fig. 1A), while the morphology of animals in the rHDGF ab only group (Fig. 1B) was not visually different from that of the normal control (Fig. 1A).

\section{Cross-Sectional Area Changes in BA}

The mean cross-sectional area of BA was $0.34 \pm 0.045$ $\mathrm{mm}^{2}$ in the control group and was $0.35 \pm 0.044 \mathrm{~mm}^{2}$ in
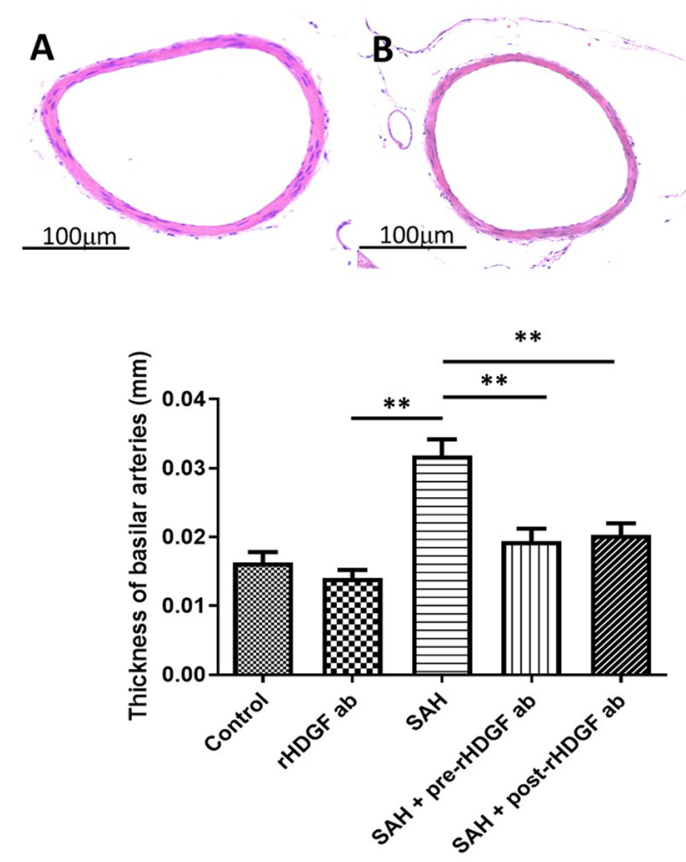

Fig. 1 Representative micrographs of BA cross sections obtained from (A) control group, (B) rHDGF ab only group, (C) SAH group, (D) $\mathrm{SAH}+$ pre-rHDGF ab group, and (E) SAH+post-rHDGF ab group. (F) Comparison of the BA cross-sectional area among control,

G
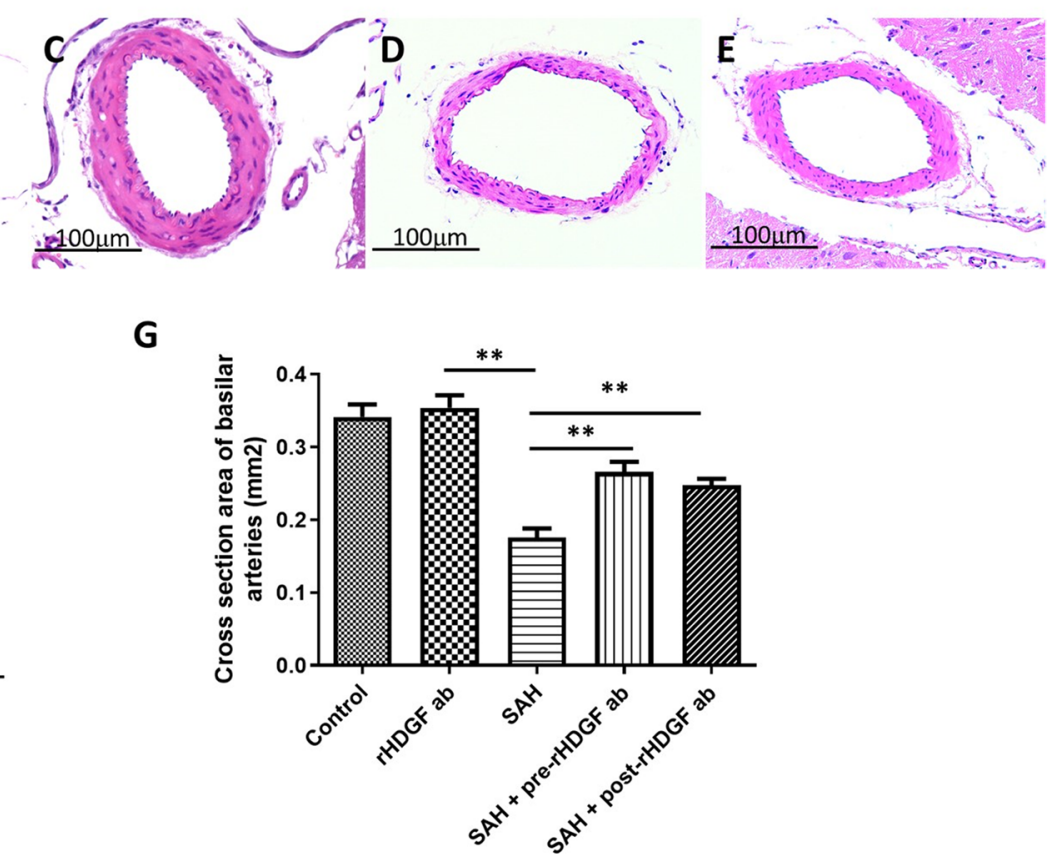

rHDGF ab, SAH, SAH + pre-rHDGF ab, and SAH + post-rHDGF ab group. (G) Comparison of the thickness of BA among the same four groups. All values are mean $\pm \mathrm{SD}(\mathrm{n}=6)$. $* * \mathrm{P}<0.01$ 
the rHDGF ab only group (Fig. 1E); there was no significant difference between these two groups. In animals with $\mathrm{SAH}$, the BA cross-sectional area $\left(0.177 \pm 0.033 \mathrm{~mm}^{2}\right)$ was reduced by $51.8 \%(\mathrm{P}<0.01)$ and $50.3 \%(\mathrm{P}<0.01)$ when compared with that in the normal control and rHDGF ab only groups, respectively. Both pre-treatment with rHDGF ab $\left(0.266 \pm 0.038 \mathrm{~mm}^{2} ; \mathrm{P}<0.05\right)$ and post-treatment with rHDGF ab $\left(0.248 \pm 0.022 \mathrm{~mm}^{2} ; \mathrm{P}<0.05\right)$ significantly increased the BA cross-sectional area when compared with that of the SAH only group (Fig. 1E).

\section{Changes in the Thickness of BA}

There was no significant difference in the thickness of BA between the normal control $(0.0163 \pm 0.004 \mathrm{~mm})$ and rHDGF ab only $(0.0140 \pm 0.003 \mathrm{~mm})$ groups (Fig. 1F). A significant increase in the thickness of BA was found in the SAH group $(0.0319 \pm 0.006 \mathrm{~mm} ; \mathrm{P}<0.01$ vs both control and rHDGF ab groups). In SAH rats pre-treated with rHDGF ab, the thickness of BA was significantly reduced $(0.0194 \pm 0.005 \mathrm{~mm} ; \mathrm{P}<0.01)$ when compared with SAH rats without treatment (Fig. 1F). In addition, In SAH rats post-treated with rHDGF ab, the thickness of BA was also significantly reduced $(0.0202 \pm 0.004 \mathrm{~mm} ; \mathrm{P}<0.01)$ when compared with SAH rats without treatment (Fig. 1F).

\section{Proliferation of Microglia and Astrocytes}

Besides in the bleeding area, activated microglia were found to diffuse into the brain parenchymal such as brain stem, cortex, and hippocampus [26, 27]. Likewise, after SAH attack, astrocytes were shown to be activated as a part of gliosis [28]. In this study, we used immunofluorescence staining for Iba- 1 and GFAP to detect the presence of microglia cells and astrocytes, respectively. Immunofluorescence staining for Iba-1 was not significantly different in the control and the rHDGF ab only groups (compare Fig. 2A and B). SAH induced proliferation of microglia cells in the rat brain (Fig. 2C, D, and E). Quantitative analysis of the intensity of Iba- 1 staining also revealed comparable levels between the control (set at 1.0) and the rHDGF ab only $(1.10 \pm 0.22)$ groups. By contrast, Iba-1
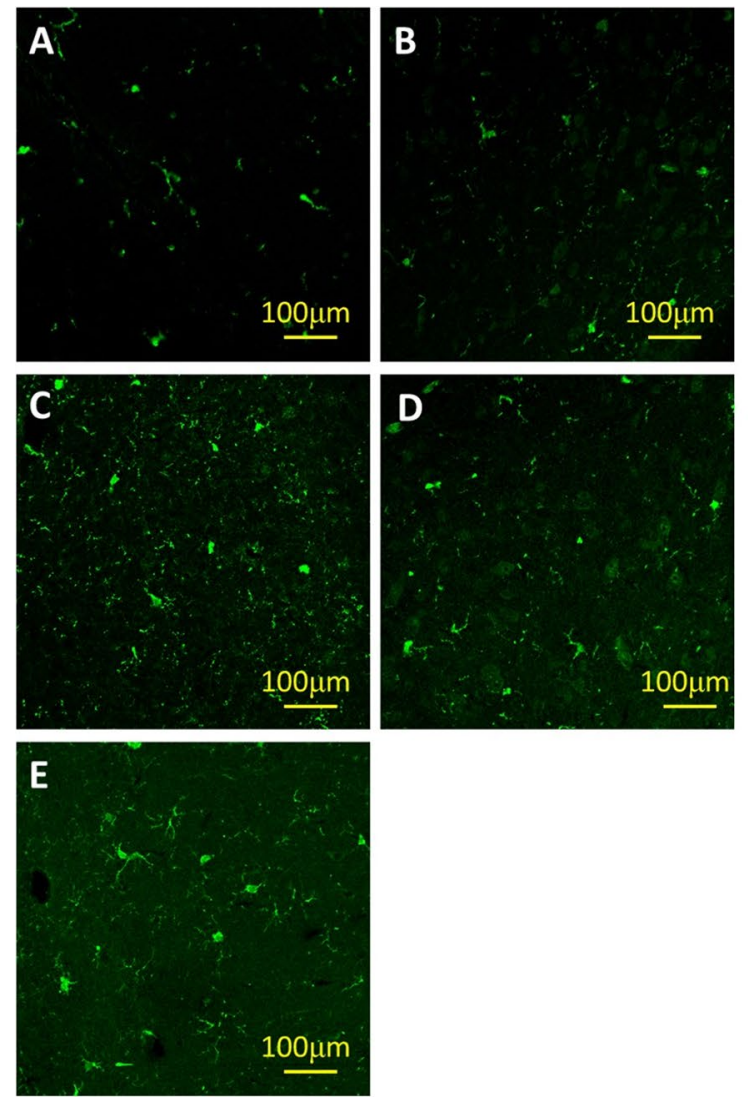

Fig. 2 Proliferation of microglia in the rat brain as determined by immunofluorescence staining for Iba-1. Representative micrographs of Iba-1 staining are shown for: (A) control group, (B) rHDGF ab only group, (C) SAH group, (D) $\mathrm{SAH}+$ pre-rHDGF ab group, and

$\mathbf{F}$

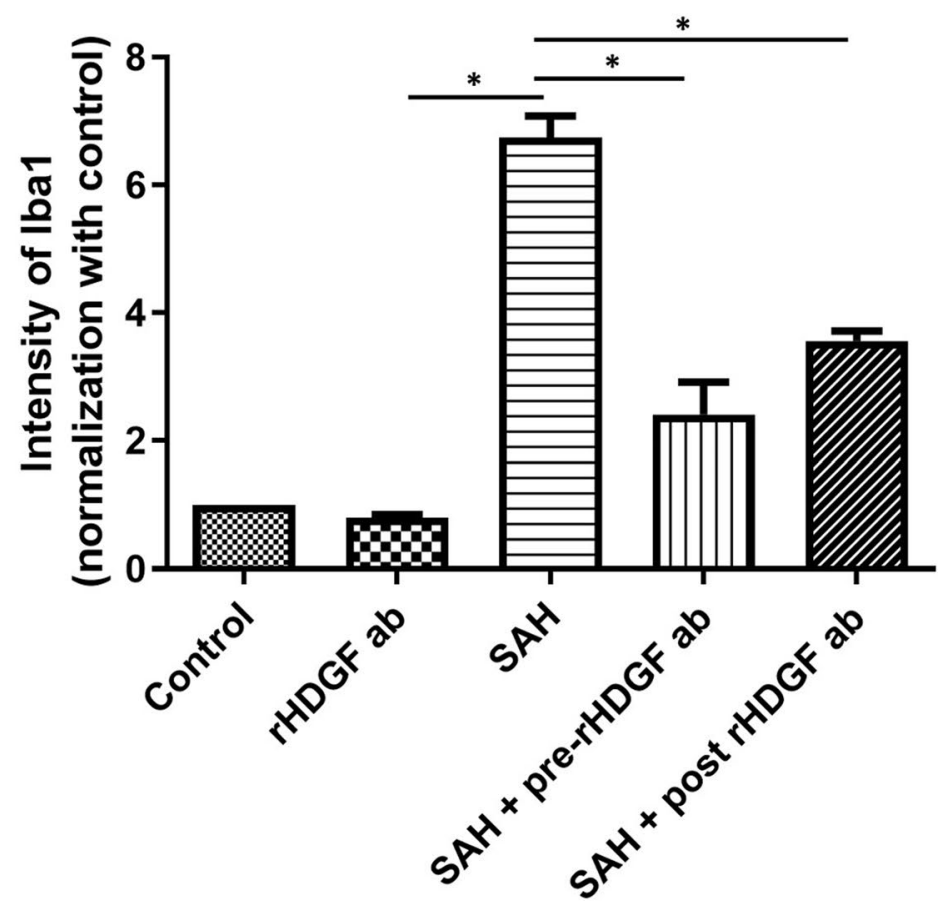

(E) SAH + post-rHDGF ab group. (F) The intensities of immunofluorescence staining in the images were quantified relative to the levels of the control animals. All values are mean $\pm \mathrm{SD}(\mathrm{n}=12) .{ }^{*} \mathrm{P}<0.05$ 
staining in the brain of the SAH rats was substantially elevated $(8.53 \pm 1.01)$, and both pre-treatment with rHDGF ab $(4.83 \pm 1.40, \mathrm{P}<0.05$ compared with the $\mathrm{SAH}$ only group) and post-treatment with rHDGF ab $(5.32 \pm 0.78, \mathrm{P}<0.05$ compared with the SAH only group) significantly reduced the proliferation of microglia cells. (Fig. 2E).

The results of immunofluorescence staining for GFAP mirrored those of the Iba-1 staining; i.e., no significant difference in GFAP staining was found between the control and the rHDGF ab only groups (compare Fig. 3A and $\mathrm{B})$, whereas $\mathrm{SAH}$ induced proliferation of astrocytes in the rat brain (Fig. 3C, D, and E). The intensities of GFAP staining the rHDGF ab only, SAH, SAH + pre-rHDGF $\mathrm{ab}$, and $\mathrm{SAH}+$ post-rHDGF ab groups were $0.81 \pm 0.08$, $6.74 \pm 0.59,2.41 \pm 0.88$, and $3.56 \pm 0.22$, respectively, relative to the value of 1.0 set for the control group. No significant difference was seen between the control and rHDGF ab only groups. The intensity of GFAP staining was significantly elevated in the SAH group $(\mathrm{P}<0.05$, compared with the control), and this value was significantly reduced in SAH rats pre-treated with $\mathrm{rHDGF}$ ab $(\mathrm{P}<0.05)$ and in SAH rats post-treated with $\mathrm{rHDGF} a b$ $(\mathrm{P}<0.05)$ (Fig. 3E).
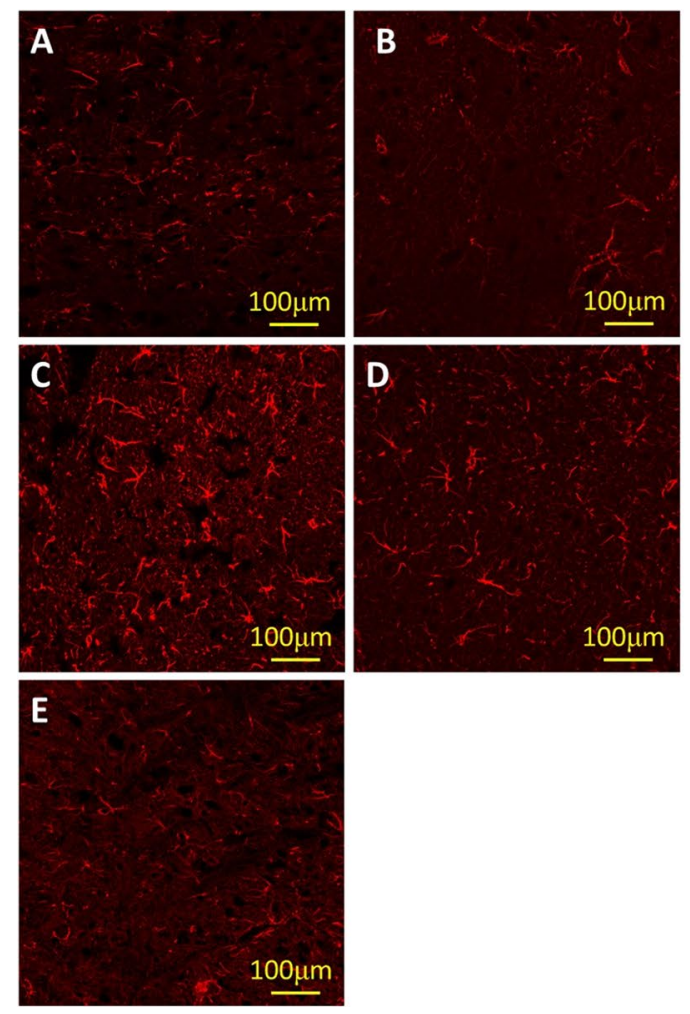

$\mathbf{F}$

Fig. 3 Proliferation of astrocytes in the rat brain as determined by immunofluorescence staining for GFAP. Representative micrographs of GFAP staining are shown for: (A) control group, (B) rHDGF ab only group, (C) SAH group, (D) SAH + pre-rHDGF ab group, and

\section{ELISA of Pro-inflammatory Factors}

To investigate the relationship between pro-inflammatory factors and SAH, the levels of TNF- $\alpha$, IL-1 $\beta$, and IL- 6 in the CSF and serum samples were examined using ELISA at $6 \mathrm{~h}$ after $\mathrm{SAH}$. No significant differences in any of the pro-inflammatory factor protein levels between the control and the rHDGF ab only groups were found in the CSF or serum samples (Fig. 4). In this early phase of SAH, expressions of TNF- $\alpha$, IL-1 $\beta$, and IL- 6 in SAH group were significantly higher than those of the rHDGF ab only group in CSF (Fig. 4). In addition, expressions of TNF- $\alpha$ and IL- $1 \beta$ in SAH group were likewise significantly higher than those of the rHDGF ab only group in serum, whereas no significant difference in the IL- 6 was seen in these samples. All of the aforementioned increases in protein expression were significantly attenuated $(\mathrm{P}<0.05$, compared with the respective SAH group upon pre-treatment with rHDGF ab and posttreatment with rHDGF ab) (Fig. 4).

\section{Western Blot Analysis}

Brain-derived neurotrophic factor (BDNF) is a molecule that regulates neuronal survival and differentiation. BDNF 

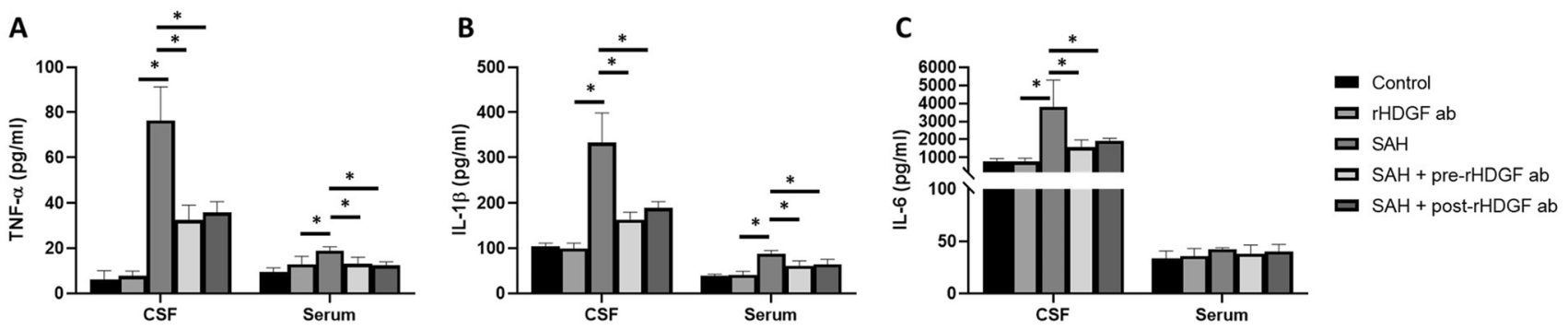

Fig. 4 ELISA assay for pro-inflammatory factors in the CSF and Serum of rats following SAH. The samples were collected at $48 \mathrm{~h}$ after SAH. The levels of $(\mathbf{A})$ TNF- $\alpha,(\mathbf{B})$ IL-1 $\beta$, and (C) IL-6 were measured using commercially available kits. All values are mean \pm SD $(n=12)$. $* P<0.05$

is initially synthesized as a precursor, proBDNF, which is trafficked to the regulated secretory pathway [29]. NF- $\mathrm{kB}$ is a nuclear transcription factor that acts as a key regulator of both inflammatory response and cell death [30,31]. Protein expressions of HDGF, proBDNF, p-NFkB (phosphorylated $\mathrm{NF \kappa B}$ ), and NFKB in the cortex were assessed at $48 \mathrm{~h}$ after $\mathrm{SAH}$. Similar to the results obtained in other measurements, no significant differences in the expression levels of these proteins between the control and the rHDGF ab only groups were found, while they were all significantly increased in the SAH group when compared with their respective controls (Fig. 5). Pre-treatment with rHDGF antibody resulted in significant decreases in the levels of proBDNF, p-NFkB/ $\mathrm{NF \kappa B}$, and HDGF when compared with the respective SAH groups (Fig. 5). In addition, post-treatment with rHDGF

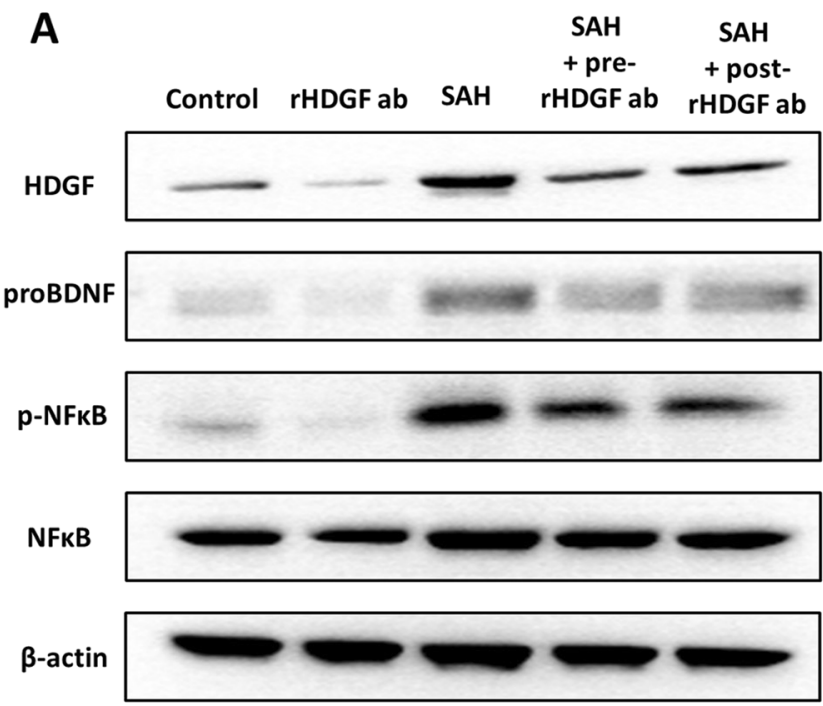

Fig. 5 Western blot analysis to show the effect of rHDGF ab pretreatment on the levels of HDGF, proBDNF, p-NFkB, and NFkB protein in the cortex of rats at $48 \mathrm{~h}$ following SAH. (A) Representative antibody also resulted in significant decreases in the levels of proBDNF, $\mathrm{p}-\mathrm{NF} \mathrm{B} / \mathrm{NF \kappa B}$, and HDGF when compared with the respective $\mathrm{SAH}$ groups.

\section{DNA Damage of Neuron Cells}

Apoptosis is a secondary response to DNA damage, and the biological goal is protecting a multicellular organism against a damaged cell [32]. In this study, immunofluorescence staining for TUNEL and neuron cells to detect the DNA damage of neuron cells. Quantitative analysis of the numbers of double immunofluorescence positive staining for TUNEL and NeuN was not significantly different in the control $(0.75 \pm 0.96)$ and the rHDGF ab $(0.50 \pm 0.58)$ only groups (Fig. 6). SAH induced the DNA damage of neuron
B

을
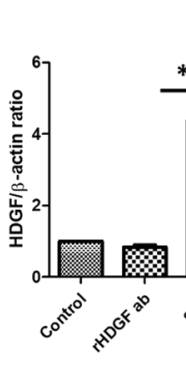

D

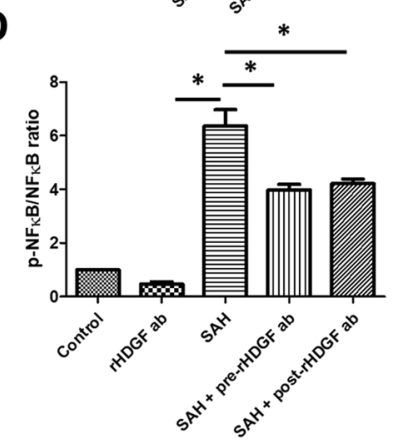

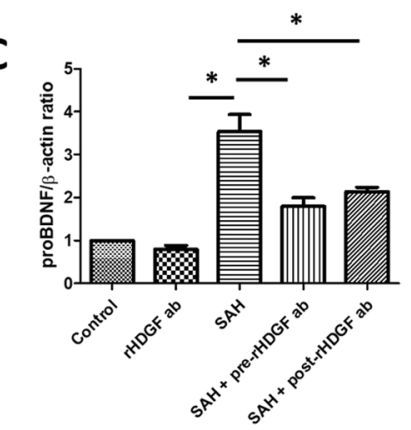

results of Western blots. The $\operatorname{HDGF}(\mathbf{B})$, proBDNF $(\mathbf{C})$, and p-NFкB/ $\mathrm{NF \kappa B}$ (D) expression levels were normalized using internal control $(\beta$-actin $)$. All values are mean \pm SD $(n=12)$ 
Fig. 6 DNA damage of neuron cells in the rat brain as determined by immunofluorescence staining for TUNEL and NeuN. Representative micrographs of immunofluorescence staining are shown for: (A) control group, rHDGF ab only group, $\mathrm{SAH}$ group, $\mathrm{SAH}+$ pre-rHDGF $\mathrm{ab}$, and SAH + post-rHDGF ab group. (B) The number of neuron cell with DNA damage from immunofluorescence staining in the images were measured in all groups. All values are mean $\pm \mathrm{SD}(\mathrm{n}=12)$. $* \mathrm{P}<0.05$

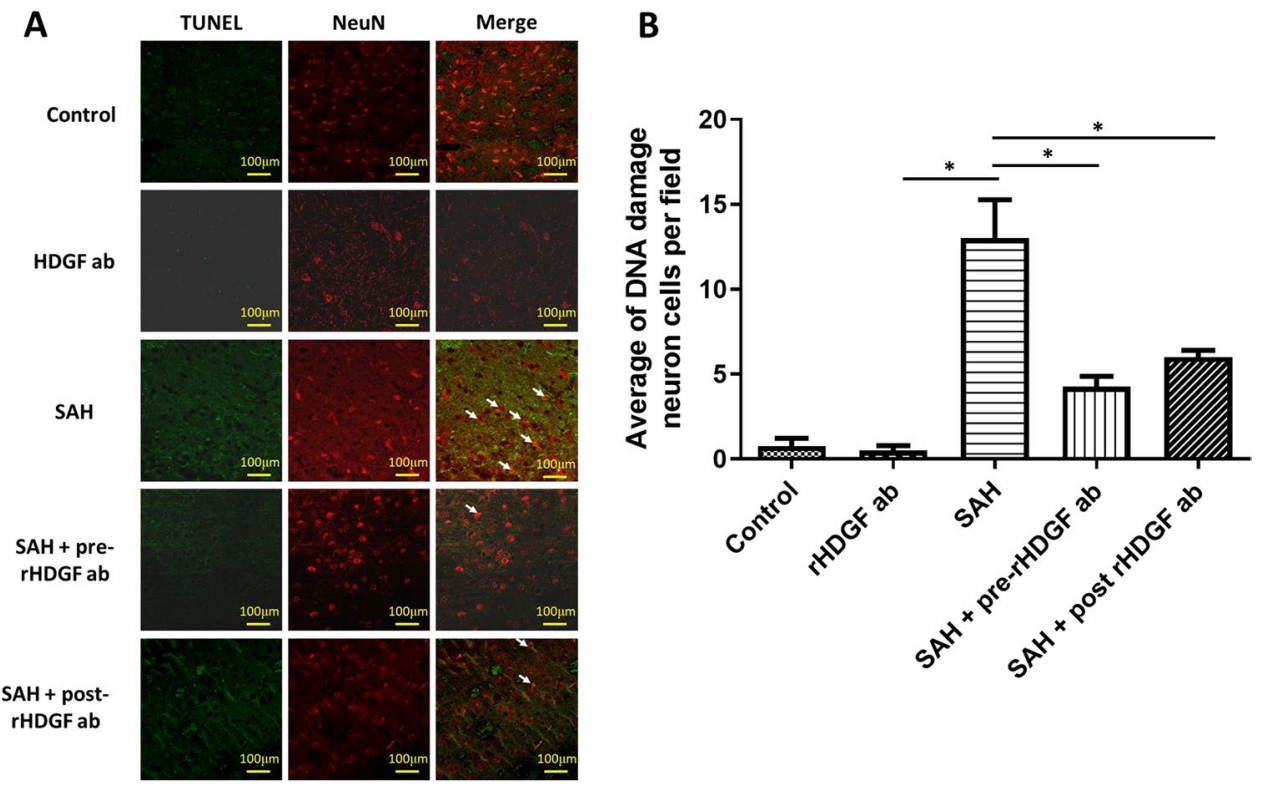

cells in the rat brain (Fig. 6A). By contrast, double staining in the brain of the SAH rats was substantially elevated $(13.00 \pm 4.55)$, and both pre-treatment with rHDGF ab $(4.25 \pm 1.26, \mathrm{P}<0.05$ compared with the $\mathrm{SAH}$ only group) and post-treatment with $\mathrm{rHDGF}$ ab $(6.00 \pm 0.707, \mathrm{P}<0.05$ compared with the SAH only group) significantly reduced the DNA damage of neuron cells (Fig. 6B).

\section{Discussion}

It has been reported that HDGF stimulates cell proliferation in fibroblasts, endothelial cells, and hepatoma cells [10] as well as mediates inflammation [33]. Consistent with these findings, our study showed that treatment with rHDGF ab could decreased the proliferation of microglia and astrocytes as well as reduced the levels of pro-inflammatory factors induced by SAH (Figs. 2, 3, and 4).

Inflammation and cytokines may participate in the pathology of blood-brain barrier (BBB) disruption and brain edema, which are characteristic features for both clinical and experimental SAH $[34,35]$. A variety of inflammatory cytokines, including IL- $1 \beta$, IL- 6 , and TNF- $\alpha$, are strongly associated with brain injury in the rat [36]. Inhibition of IL-1 $\beta$ has been shown to attenuate early brain injury (EBI) and improve BBB function after SAH [37]. The present study showed that pre-treatment with rHDGF ab decreased the SAH-induced production of TNF- $\alpha$, IL-1 $\beta$, and IL- 6 .

Many studies have shown that HDGF may play a role in apoptosis. For example, silencing the HDGF gene has been demonstrated to prevent TNF- $\alpha /$ cycloheximide-induced apoptosis [38]. In addition, HDGF is found to be essential for TNF $\alpha$-induced release of pro-apoptotic factors from mitochondria [38]. In contrast, it has been demonstrated that knockdown of HDGF gene induces apoptosis [39, 40] and cell cycle arrest in several human cancers. HDGF knockdown not only induces expression and de-phosphorylation of the pro-apoptotic protein Bad, but also inactivates ERK and Akt, resulting in activation of the intrinsic apoptotic pathway in cancers [40-42]. However, in our study, blocking HDGF attenuated SAH-induced neuron cell apoptosis in the brain (Fig. 6). Similarly, HDGF knockdown triggers the Fas-mediated extrinsic apoptotic pathway in HepG2 cells through the nuclear factor- $\kappa B$ $(\mathrm{NF}-\kappa \mathrm{B})$ signaling [43]. NF- $\mathrm{B}$ is a nuclear transcription factor that acts as a key regulator of both inflammatory response and cell death $[30,31]$. Our study showed that pre-treatment with rHDGF ab down-regulated inflammatory factors and $\mathrm{p}-\mathrm{NF} \mathrm{B} / \mathrm{NF} \kappa \mathrm{B}$ ratio. These data supported the notion that blocking HDGF attenuated apoptosis of neuron cells in the cortex after SAH (Fig. 5D).

Brain-derived neurotrophic factor (BDNF), a molecule that regulates neuronal survival and differentiation, has a critical role in synaptic plasticity [44]. However, BDNF is initially synthesized as a precursor, proBDNF, which is trafficked to the regulated secretory pathway [29]. A previous report showed that mature BDNF can protect neurons from amyloid-beta $(\mathrm{A} \beta)$-induced apoptosis by increasing the expression of bcl-2 [36]. (Irrelevant) Our results showed that the protein levels of proBDNF were significantly elevated in the cortex of SAH rats, and pretreatment with rHDGF ab decreased proBDNF protein expression following SAH, suggesting that the beneficial effects seen with blocking HDGF may be mediated, at least partially, by the BDNF pathway. 


\section{Conclusion}

This is the first study to investigate the relationship between HDGF and SAH. In our results, HDGF induced inflammation and neuron cell apoptosis after SAH. Blocking HDGF reduced SAH-induced vasospasm and attenuated inflammation and cell apoptosis possibly through inhibiting proBDNF and $\mathrm{p}-\mathrm{NF} \kappa \mathrm{B} / \mathrm{NF} \kappa \mathrm{B}$ pathways.

Acknowledgements We thank the Center for Research Resources and Development of Kaohsiung Medical University in immunofluorescence staining using LSM 700 Confocal Microscope and the Center for Medical Informatics and Statistics of Kaohsiung Medical University for providing administrative and funding support.

Author Contribution Chia-Li Chung wrote this MS.

Chieh-Hsin Wu performed the statistical analysis.

Yu-Hua Huang performed the statistical analysis.

Shu-Chuan Wu made animal model.

Chee-Yin Chai performed immunofluorescence staining.

Hung-Pei Tsai revised the manuscript.

Aij-Lie Kwan revised the manuscript.

Funding This study was supported by grants from the Kaohsiung Medical University Hospital (KMUH108-8M26, KMUH107-M701, and KMUH106-M618).

Data Availability Not applicable.

Code Availability Not applicable.

\section{Declarations}

Ethics Approval Not applicable.

Human and Animal Rights Consent The animal use protocol listed below has been reviewed and approved by the Institutional Animal Care and Use Committee (IACUC) (105258).

Consent to Participate Not applicable.

Consent for Publication Not applicable.

Conflict of Interest The authors declare no competing interests.

Open Access This article is licensed under a Creative Commons Attribution 4.0 International License, which permits use, sharing, adaptation, distribution and reproduction in any medium or format, as long as you give appropriate credit to the original author(s) and the source, provide a link to the Creative Commons licence, and indicate if changes were made. The images or other third party material in this article are included in the article's Creative Commons licence, unless indicated otherwise in a credit line to the material. If material is not included in the article's Creative Commons licence and your intended use is not permitted by statutory regulation or exceeds the permitted use, you will need to obtain permission directly from the copyright holder. To view a copy of this licence, visit http://creativecommons.org/licenses/by/4.0/.

\section{References}

1. Bederson JB, Connolly ES Jr, Batjer HH, Dacey RG, Dion JE, Diringer MN, et al. Guidelines for the management of aneurysmal subarachnoid hemorrhage: a statement for healthcare professionals from a special writing group of the Stroke Council American Heart Association. Stroke. 2009;40:994-1025.

2. Alaraj A, Charbel FT, Amin-Hanjani S. Peri-operative measures for treatment and prevention of cerebral vasospasm following subarachnoid hemorrhage. Neurol Res. 2009;31:651-9.

3. Kassell NF, Sasaki T, Colohan AR, Nazar G. Cerebral vasospasm following aneurysmal subarachnoid hemorrhage. Stroke. 1985;16:562-72.

4. Kongkathip N, Kongkathip B, Siripong P, Sangma C, Luangkamin S, Niyomdecha M, et al. Potent antitumor activity of synthetic 1,2-naphthoquinones and 1,4-naphthoquinones. Bioorg Med Chem. 2003;11:3179-91.

5. Chowdhary RK, Shariff I, Dolphin D. Drug release characteristics of lipid based benzoporphyrin derivative. J Pharm Pharm Sci. 2003;6:13-9.

6. Al-Khindi T, Macdonald RL, Schweizer TA. Cognitive and functional outcome after aneurysmal subarachnoid hemorrhage. Stroke. 2010;41:e519-36.

7. Friedrich V, Flores R, Sehba FA. Cell death starts early after subarachnoid hemorrhage. Neurosci Lett. 2012;512:6-11.

8. Claassen J, Bernardini GL, Kreiter K, Bates J, Du YE, Copeland D, et al. Effect of cisternal and ventricular blood on risk of delayed cerebral ischemia after subarachnoid hemorrhage: the Fisher scale revisited. Stroke. 2001;32:2012-20.

9. Nakamura H, Izumoto $Y$, Kambe H, Kuroda T, Mori T, Kawamura K, et al. Molecular cloning of complementary DNA for a novel human hepatoma-derived growth factor. Its homology with high mobility group-1 protein. J Biol Chem. 1994;269:25143-9.

10. Bremer S, Klein K, Sedlmaier A, Abouzied M, Gieselmann V, Franken S. Hepatoma-derived growth factor and nucleolin exist in the same ribonucleoprotein complex. BMC Biochem. 2013;14:2.

11. Kishima Y, Yoshida K, Enomoto H, Yamamoto M, Kuroda T, Okuda Y, et al. Antisense oligonucleotides of hepatoma-derived growth factor (HDGF) suppress the proliferation of hepatoma cells. Hepatogastroenterology. 2002;49:1639-44.

12. Enomoto H, Yoshida K, Kishima Y, Kinoshita T, Yamamoto $\mathrm{M}$, Everett AD, et al. Hepatoma-derived growth factor is highly expressed in developing liver and promotes fetal hepatocyte proliferation. Hepatology. 2002;36:1519-27.

13. Enomoto H, Yoshida K, Kishima Y, Okuda Y, Nakamura H. Participation of hepatoma-derived growth factor in the regulation of fetal hepatocyte proliferation. J Gastroenterol. 2002;37(Suppl 14):158-61.

14. Enomoto H, Nakamura H, Liu W, Yoshida K, Okuda Y, Imanishi $\mathrm{H}$, et al. Hepatoma-derived growth factor is induced in liver regeneration. Hepatol Res. 2009;39:988-97.

15. Everett AD, Narron JV, Stoops T, Nakamura H, Tucker A. Hepatoma-derived growth factor is a pulmonary endothelial cellexpressed angiogenic factor. Am J Physiol Lung Cell Mol Physiol. 2004;286:L1194-201.

16 Cilley RE, Zgleszewski SE, Chinoy MR. Fetal lung development: airway pressure enhances the expression of developmental genes. J Pediatr Surg. 2000;35:113-8 (discussion 9).

17. Oliver JA, Al-Awqati Q. An endothelial growth factor involved in rat renal development. J Clin Invest. 1998;102:1208-19.

18. Everett $\mathrm{AD}$. Identification, cloning, and developmental expression of hepatoma-derived growth factor in the developing rat heart. Dev Dyn. 2001;222:450-8. 
19. Narron JV, Stoops TD, Barringhaus K, Matsumura M, Everett AD. Hepatoma-derived growth factor is expressed after vascular injury in the rat and stimulates smooth muscle cell migration. Pediatr Res. 2006;59:778-83.

20. Everett AD, Stoops T, McNamara CA. Nuclear targeting is required for hepatoma-derived growth factor-stimulated mitogenesis in vascular smooth muscle cells. J Biol Chem. 2001;276:37564-8.

21. Everett AD, Lobe DR, Matsumura ME, Nakamura H, McNamara CA. Hepatoma-derived growth factor stimulates smooth muscle cell growth and is expressed in vascular development. J Clin Invest. 2000;105:567-75.

22. Hu TH, Huang CC, Liu LF, Lin PR, Liu SY, Chang HW, et al. Expression of hepatoma-derived growth factor in hepatocellular carcinoma. Cancer. 2003;98:1444-56.

23. Yamamoto S, Tomita Y, Hoshida Y, Takiguchi S, Fujiwara Y, Yasuda T, et al. Expression of hepatoma-derived growth factor is correlated with lymph node metastasis and prognosis of gastric carcinoma. Clin Cancer Res. 2006;12:117-22.

24. Huang YH, Chung CL, Tsai HP, Wu SC, Chang CZ, Chai $\mathrm{CY}$, et al. Hyperglycemia aggravates cerebral vasospasm after subarachnoid hemorrhage in a rat model. Neurosurgery. 2017;80:809-15.

25. Jeon H, Ai J, Sabri M, Tariq A, Shang X, Chen G, et al. Neurological and neurobehavioral assessment of experimental subarachnoid hemorrhage. BMC Neurosci. 2009;10:103.

26. Provencio JJ, Vora N. Subarachnoid hemorrhage and inflammation: bench to bedside and back. Semin Neurol. 2005;25:435-44.

27. Huang LT, Li H, Sun Q, Liu M, Li WD, Li S, et al. IL-33 expression in the cerebral cortex following experimental subarachnoid hemorrhage in rats. Cell Mol Neurobiol. 2015;35:493-501.

28. van Dijk BJ, Vergouwen MD, Kelfkens MM, Rinkel GJ, Hol EM. Glial cell response after aneurysmal subarachnoid hemorrhage functional consequences and clinical implications. Biochim Biophys Acta. 2016;1862:492-505.

29. Chen ZY, Ieraci A, Teng H, Dall H, Meng CX, Herrera DG, et al. Sortilin controls intracellular sorting of brain-derived neurotrophic factor to the regulated secretory pathway. J Neurosci. 2005;25:6156-66.

30. Mattson MP, Camandola S. NF-kappaB in neuronal plasticity and neurodegenerative disorders. J Clin Invest. 2001;107:247-54.

31. Pizzi M, Sarnico I, Lanzillotta A, Battistin L, Spano P. Postischemic brain damage: NF-kappaB dimer heterogeneity as a molecular determinant of neuron vulnerability. FEBS J. 2009;276:27-35.

32. Wang JY. DNA damage and apoptosis. Cell Death Differ. 2001;8:1047-8.

33. Wang EM, Hu TH, Huang CC, Chang YC, Yang SM, Huang ST, et al. Hepatoma-derived growth factor participates in concanavalin A-induced hepatitis. FASEB J. 2020;34:16163-78.
34. Zetterling M, Hallberg L, Hillered L, Karlsson T, Enblad P, Ronne EE. Brain energy metabolism in patients with spontaneous subarachnoid hemorrhage and global cerebral edema. Neurosurgery. 2010;66:1102-10.

35. Altay O, Suzuki H, Hasegawa Y, Caner B, Krafft PR, Fujii M, et al. Isoflurane attenuates blood-brain barrier disruption in ipsilateral hemisphere after subarachnoid hemorrhage in mice. Stroke. 2012;43:2513-6.

36. Liu Z, Ma D, Feng G, Ma Y, Hu H. Recombinant AAV-mediated expression of human BDNF protects neurons against cell apoptosis in Abeta-induced neuronal damage model. J Huazhong Univ Sci Technolog Med Sci. 2007;27:233-6.

37. Sozen T, Tsuchiyama R, Hasegawa Y, Suzuki H, Jadhav V, Nishizawa $S$, et al. Role of interleukin-1beta in early brain injury after subarachnoid hemorrhage in mice. Stroke. 2009;40:2519-25.

38. Machuy N, Thiede B, Rajalingam K, Dimmler C, Thieck O, Meyer $\mathrm{TF}$, et al. A global approach combining proteome analysis and phenotypic screening with RNA interference yields novel apoptosis regulators. Mol Cell Proteomics. 2005;4:44-55.

39. Yu Y, Shen H, Yu H, Zhong F, Zhang Y, Zhang C, et al. Systematic proteomic analysis of human hepotacellular carcinoma cells reveals molecular pathways and networks involved in metastasis. Mol Biosyst. 2011;7:1908-16.

40. Hsu SS, Chen CH, Liu GS, Tai MH, Wang JS, Wu JC, et al. Tumorigenesis and prognostic role of hepatoma-derived growth factor in human gliomas. J Neurooncol. 2012;107:101-9.

41. Ooi BN, Mukhopadhyay A, Masilamani J, Do DV, Lim CP, Cao $\mathrm{XM}$, et al. Hepatoma-derived growth factor and its role in keloid pathogenesis. J Cell Mol Med. 2010;14:1328-37.

42. Tsang TY, Tang WY, Tsang WP, Co NN, Kong SK, Kwok TT. Downregulation of hepatoma-derived growth factor activates the Bad-mediated apoptotic pathway in human cancer cells. Apoptosis. 2008;13:1135-47.

43. Tsang TY, Tang WY, Tsang WP, Co NN, Kong SK, Kwok TT. Mechanistic study on growth suppression and apoptosis induction by targeting hepatoma-derived growth factor in human hepatocellular carcinoma HepG2 cells. Cell Physiol Biochem. 2009;24:253-62.

44. Ma L, Wang DD, Zhang TY, Yu H, Wang Y, Huang SH, et al. Region-specific involvement of BDNF secretion and synthesis in conditioned taste aversion memory formation. J Neurosci. 2011;31:2079-90.

Publisher's Note Springer Nature remains neutral with regard to jurisdictional claims in published maps and institutional affiliations. 


\section{Authors and Affiliations}

\section{Chia-Li Chung ${ }^{1,2} \cdot$ Chieh-Hsin Wu ${ }^{3,4} \cdot$ Yu-Hua Huang ${ }^{5} \cdot$ Shu-Chuan $\mathrm{Wu}^{3} \cdot$ Chee-Yin Chai ${ }^{6,7} \cdot$ Hung-Pei Tsai ${ }^{3,8} \odot$. Aij-Lie Kwan ${ }^{3,4,8,9}$}

1 Graduate Institute of Medicine, College of Medicine, Kaohsiung Medical University, Kaohsiung, Taiwan

2 Department of Surgery, Kaohsiung Municipal Siaogang Hospital, Kaohsiung, Taiwan

3 Department of Neurosurgery, Kaohsiung Medical University Hospital, Kaohsiung, Taiwan

4 Department of Surgery, College of Medicine, Kaohsiung Medical University, Kaohsiung, Taiwan

5 Department of Neurosurgery, Kaohsiung Chang Gung Memorial Hospital and Chang Gung University College of Medicine, Kaohsiung, Taiwan
6 Department of Pathology, Kaohsiung Medical University Hospital, Kaohsiung, Taiwan

7 Department of Pathology, College of Medicine, Kaohsiung Medical University, Kaohsiung, Taiwan

8 Division of Neurosurgery, Department of Surgery, Kaohsiung Medical University Hospital, Kaohsiung Medical University, No.100, Tzyou 1st Road, Kaohsiung 80756, Taiwan

9 Department of Neurosurgery, University of Virginia, Charlottesville, VA, USA 